\title{
Abschied ohne Schrecken
}

\author{
2018 soll endgültig Schluss sein mit dem seit Jahrzehnten bewähr- \\ ten ISDN-Netz. Dann wollen die Telekom, 1\&1 und andere große \\ Telefonnetzanbieter ihre Leitungen endgültig auf "All-IP“ umstel- \\ len. Gerade gewerbliche Kunden wie Arztpraxen kann es auch \\ schon früher treffen. Welche Optionen haben Betroffene?
}

Gut 20 Jahre lang hat ISDN (Integrated Services Digital Network) treue Dienste geleistet. Doch nun wollen die Telekommunikationsanbieter ISDN loswerden. Spätestens 2018 plant Marktführer Telekom, die letzten alten Anschlüsse abgeschaltet zu haben. Auch Telekom-Wiederverkäufer wie $1 \& 1$ sind betroffen. Nur Vodafone will seinen Geschäftskunden notfalls noch ein paar Jahre länger Zeit lassen. Doch auch bei diesem Netzbetreiber gilt ISDN als klares Auslaufmodell. Der Hintergrund: Für die Anbieter werden Betrieb und Unterhalt der ISDNTechnik immer teurer. Sie möchten die Kundenanschlüsse deshalb lieber auf die günstigere und modernere IP-Technik umstellen - vor allem, wenn sie mit DSLInternetleitungen kombiniert sind.

\section{Umstellung läuft auf Hochtouren}

Der neue Anschlusstyp nutzt zum Telefonieren das Internetprotokoll („IP“). Mit zwei Leitungen und drei bis zehn Rufnummern bietet er auch vergleichbaren Komfort wie ISDN. Und während IPTelefonie in früheren Jahren zu Recht den Ruf hatte, fehleranfällig und qualitativ minderwertig zu sein, ist die Technik

\section{All-IP - was ist das?}

- Der Begriff bezeichnet die Umstellung bisheriger Übertragungstechniken in Telekommunikationsnetzen auf die Basis des Internetprotokolls (IP) - also vom analogen auf den rein digitalen Anschluss.

- Einzelne Dienste wie Telefonie, Fax, Mobilfunk, Internet oder Fernsehen werden dabei einheitlich über die digitalen Leitungen geschickt.

- Der Nachteil: Fällt die DSL-Leitung aus oder der Router geht kaputt beziehungsweise hat eine Störung, dann schweigt auch das Telefon. mittlerweile deutlich gereift. Sie bietet heute fast dieselbe Zuverlässigkeit und Qualität wie das alte ISDN. Daher läuft die Umstellung bereits auf vollen Touren.

Viele ISDN-Kunden wurden von ihrem Anbieter schon angeschrieben und zum Wechsel gedrängt.

\section{Rechtzeitig reagieren}

Kein Telefon und kein Internet mehr zu haben, wäre für Geschäftskunden natürlich der GAU. Deshalb sollten Betroffene sich nicht allzu viel Zeit lassen, sich auf die neue Sachlage einzustellen.

Wie man angesichts der „IP-Umstellung “ am besten vorgehen sollte, hängt von der bislang vorhandenen Telefontechnik ab. Größere Praxen oder Kliniken, die eine ausgewachsene Nebenstellenanlage betreiben, wenden sich am besten an den Anbieter oder Betreuer dieser Systeme. Erfreulich: In den meisten Fällen muss nicht die komplette Telefonanlage ausgewechselt werden. Oft genügt es, die Komponente $\mathrm{zu}$ modernisieren, die bisher den Anschluss an das ISDN verwaltete. Doch neben den Telefonanschlüssen versorgen ISDN-Leitungen oft auch weitere Systeme - EC-Bezahlterminals, Alarmanlagen oder Aufzugnotrufsysteme. Auch hier gilt: Ansprechpartner für Fragen zur IP-Umstellung ist der jeweilige Anbieter.

Einzelpraxen und kleine Praxisgemeinschaften haben in der Regel keine Telefonanlage, ihnen genügen häufig ein bis zwei ISDN-Anschlüsse mit zwei bis vier Außenleitungen. Dementsprechend gibt es oft auch gar kein Wartungsunternehmen, das für die installierte Technik zuständig wäre. „Hilf Dir selbst“ ist hier dann angesagt.

\section{Router als Adapter und Telefonanlage}

Wenn nun die externe Telefonleitung auf IP-Technik umgestellt wird, müssen die internen Telefone und andere Geräte wie Fax oder Anrufbeantworter nicht unbedingt ersetzt werden. Benötigt wird vielmehr ein Adapter, der zwischen der intern genutzten, vorhandenen ISDNTechnik und der künftigen externen IP-Leitung „übersetzt“. Diese Funktion übernehmen nicht zuletzt moderne Internet-Router. Insbesondere die Topmodelle der „Fritzboxen“ des Berliner Anbieters AVM beherrschen diese Übung. Sie verfügen zur Teilnehmerseite über eine ISDN-Schnittstelle (Fachjargon: S0-Bus), an der sich vorhandene ISDNGeräte einfach anschließen lassen. In Richtung Telefonnetz kommunizieren sie per IP. Anbieter wie 1\&1 und Vodafone liefern dieselben AVM-Router zum Teil unter eigenen Produktbezeichnungen.

Nicht ganz so einfach ist der Umstieg bei den Speedport-Routern, die die Telekom ihren Kunden anpreist, denn sie verfügen nur über analoge Telefonschnittstellen. Mit dem für $70 €$ separat angebotenen "Speedport ISDN-Adapter“ lassen sich aber auch Telekom-Router entsprechend erweitern.

\section{Anbieter an Kosten beteiligen}

Übrigens: Wenn durch die IP-Umstellung Kosten für Neuanschaffungen oder Anpassungen anfallen, sollte man auf jeden Fall den Netzbetreiber darauf ansprechen. Zumindest anteilig werden solche Kosten im Rahmen der Kundenpflege nicht selten übernommen.

Und noch eine gute Nachricht: Oft steht nach der Umstellung sogar etwas mehr Telefonkomfort zur Verfügung als vorher. So sind in den genannten Routern vollwertige, kleine Telefonanlagen integriert, die Funktionen wie Makeln, Rückfragen oder Konferenzschaltungen unterstützen. Bei Bedarf lassen sich an den Internetboxen sogar schnurlose Telefone anmelden. Und im Zusammenspiel mit geeigneten Endgeräten sowie Gegenstellen lässt sich dann unter Umständen sogar die neue „HD-Telefonie“ nutzen. Sie liefert noch höhere Sprachqualität und Verständlichkeit als das bisherige ISDN. Das wäre zumindest ein kleiner Lohn der Mühen und Kosten der Umstellung. 